\title{
RAMAN SCATTERING FROM PHONONS AND MAGNONS IN ANTIFERROMAGNETIC $\mathrm{Fe}_{3} \mathrm{BO}_{6}$
}

\author{
H. Navarro*, J.E. Potts ${ }^{\dagger}$ and R. Merlin \\ Department of Physics, University of Michigan, Ann Arbor, MI 48109, U.S.A.
}

(Received 20 October 1983 by H. Suhl)

\begin{abstract}
Raman scattering by phonons and by magnon pairs has been observed in $\mathrm{Fe}_{3} \mathrm{BO}_{6}$. Of the predicted 60 Raman-active modes, 39 have been identıfied and classified according to their symmetries. The two-magnon band shows a strong decrease in intensity with increasing temperature, and almost vanishes close to $T_{N}=508 \mathrm{~K}$. The origin of this effect is attributed to the existence of a nearly dispersionless magnon branch.
\end{abstract}

$\mathrm{Fe}_{3} \mathrm{BO}_{6}$ IS A CANTED antiferromagnet $\left(T_{N}=508 \mathrm{~K}\right)$ [1] which crystallizes in the orthorhombic $D_{2 h}^{16}(P n m a)$ structure with four formula units per unit cell [2]. The Fe spins couple ferromagnetically within (001) planes and, in successive $(001)$ planes, they are oriented $\uparrow \downarrow$ [3]. At $T_{S R}=415 \mathrm{~K}$, a sudden spin reorientation takes place where the weak ferromagnetic moment rotates by $90^{\circ}$ [3]. Depending on the temperature, the spins are almost parallel to either an equivalent [001] (for $T<T_{S R}$ ) or [100] direction $\left(T_{S R}<T<T_{N}\right)[3]$.

To our knowledge, no study of the phonon spectrum of $\mathrm{Fe}_{3} \mathrm{BO}_{6}$ has been previously reported.

Reference to the magnon structure is found in the optical absorption data which shows the presence of a magnon (hot) sideband $419 \mathrm{~cm}^{-1}$ below the $I|0,3 / 2\rangle$ exciton [4]. Five optical magnon branches are expected in $\mathrm{Fe}_{3} \mathrm{BO}_{6}$. As shown below, the branch involved in the optical process gives the strongest contribution to twomagnon scattering.

The sample for this study was a single crystal, $3 \times 3 \times 1 \mathrm{~mm}$, grown by chemical transport. Because $\mathrm{Fe}_{3} \mathrm{BO}_{6}$ is quite opaque in the visible $\left(\alpha \simeq 10^{4}-10^{5}\right.$ $\mathrm{cm}^{-1}$ ) [4], the Raman measurements were made in the backscattering geometry using the $6471 \AA$ line of a $\mathrm{Kr}^{+}$-laser; incident power levels were kept low $(\leqslant 50$ $\mathrm{mW}$ ) to prevent sample heating. A Spex 1403 double monochromator and a RCA 31034A photomultiplier were used for detecting the scattered light.

Site group analysis [5] shows that the allowed Raman modes in $\mathrm{Fe}_{3} \mathrm{BO}_{6}$ transform as $17 A_{\mathrm{g}}+13 B_{1 \mathrm{~g}}+$ $17 B_{2 g}+13 B_{3 g}$. We have identified 39 of the predicted

\footnotetext{
* Permanent address: Departmento de Fisica. Universidad Autónoma de Puebla, Apdo. Postal J-48, Puebla, Pue-México.

$\dagger$ Permanent address: Department of Natural Sciences, University of Michigan-Dearborn, Dearborn, MI 48128, U.S.A.
}

60 active phonons and listed the room-temperature energies in Table 1. None of these modes exhibits any unusual temperature dependence of its frequency or intensity which might be correlated with the magnetic transitions. The feature that we assign to the scattering by a magnon pair occurs in the $x(z z) \bar{x} A_{g}$-spectrum at $742 \mathrm{~cm}^{-1}(300 \mathrm{~K})$. It is shown at temperature below and above $T_{N}$ in Fig. 1, which also shows the $616 \mathrm{~cm}^{-1}$ $A_{\mathrm{g}}$-phonon. Our assignment of the $742 \mathrm{~cm}^{-1}$-peak as due to two-magnon scattering is based, first, on the data of Fig. 2 which shows the temperature dependence of the integrated intensity of this structure. The rapid decrease in intensity and the near disappearance at, or just above, $T_{N}$ is a strong indication that the scattering involves a magnetic excitation. In addition, the value of $742 \mathrm{~cm}^{-1}$ is only $\approx 10 \%$ less than twice the energy of the magnon determined from the optical data [4]. It is well known that magnon-magnon interactions play an essential role in determining the structure of twomagnon spectra [5]. This is because the magnons created in the scattering process are close together in real space and, therefore, interact strongly. We attribute the $10 \%$ difference to the renormalization of magnon-pair frequencies due to this effect and, consequently, we ascribe our peak and the magnon-sideband [4] to the same magnon branch.

Further, although indirect, support for our assignment is suggested by a comparison with the case of hematite $\left(\alpha-\mathrm{Fe}_{2} \mathrm{O}_{3}\right)$ which also exhibits a two-magnon structure with intensity approaching zero in the vicinity of $T_{N}$ [7]. The two-magnon band in $\alpha-\mathrm{Fe}_{2} \mathrm{O}_{3}$ 1s extremely narrow $\left(\Gamma / \Omega \approx 0.03\right.$ at $T=0.08 T_{N} ; \Gamma$ is the FWHM and $\Omega$ the peak position) and shifts toward lower energies by less than $2 \%$ when $T$ goes from 0.08 $T_{N}$ to $0.75 T_{N}$ [7]. As shown in Fig. 3, the corresponding values for $\mathrm{Fe}_{3} \mathrm{BO}_{6}$ are comparable: $\Gamma / \Omega \approx 0.01$ at $T=0.2 T_{N}$ and a shift of $\approx 3 \%$ in the temperature range $0.2 T_{N}<T<1.1 T_{N}$. The narrow width in $\alpha-\mathrm{Fe}_{2} \mathrm{O}_{3}$ 
Table 1. Energies of the 39 observed optical phonons (in units of $\mathrm{cm}^{-1}$ ) at $\mathrm{T}=300 \mathrm{~K}$

\begin{tabular}{llll}
\hline$A_{g}$ & $B_{1 g}$ & $B_{2 g}$ & $B_{3 g}$ \\
\hline 124,166 & 164 & 150,184 & $114,154,178$ \\
$204,240,294$ & 218,260 & 221 & \\
$320,332,388$ & 382 & 318,390 & 386 \\
400 & 428,470 & 418,448 & 408,456 \\
508 & 592 & & \\
616 & & 610 & 630 \\
714,742 & & & \\
822 & & 890 & \\
986 & 910 & & \\
\hline
\end{tabular}

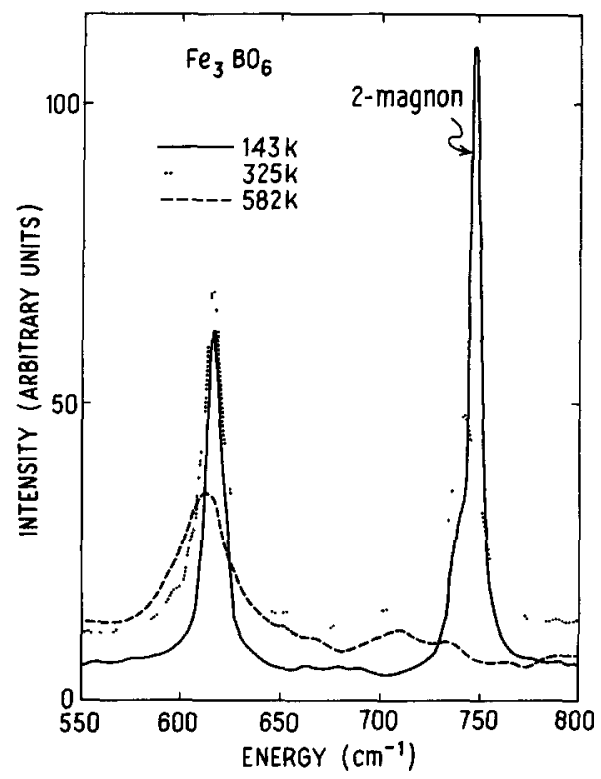

Fig. 1. Spectra of $\mathrm{Fe}_{3} \mathrm{BO}_{6}$ for different temperatures showing the two-magnon peak and the $A_{g}$-phonon at $616 \mathrm{~cm}^{-1}$.

originates in the nearly dispersionless optical-magnon branch [8], as determined by neutron scattering measurements [9]. Based on the similar properties of the Raman data for the two materials, we conclude that an almost dispersionless branch should also occur in $\mathrm{Fe}_{3} \mathrm{BO}_{6}$.

The $\mathrm{Fe}$ spins in $\alpha-\mathrm{Fe}_{2} \mathrm{O}_{3}$ are strongly coupled to 12 nearest neighbors ( $\mathrm{nn}$ ) that belong to the opposite sublattice but only weakly coupled to a single nn with the same spin orientation [10]. Such a tightly-coupled cluster of spins leads to essentially localized excitations and, consequently, to a flat magnon branch. The source of localization in $\mathrm{Fe}_{3} \mathrm{BO}_{6}$ is not apparent as the exchange constants of this material are not yet available. A possibility is that the clusters are made out of just

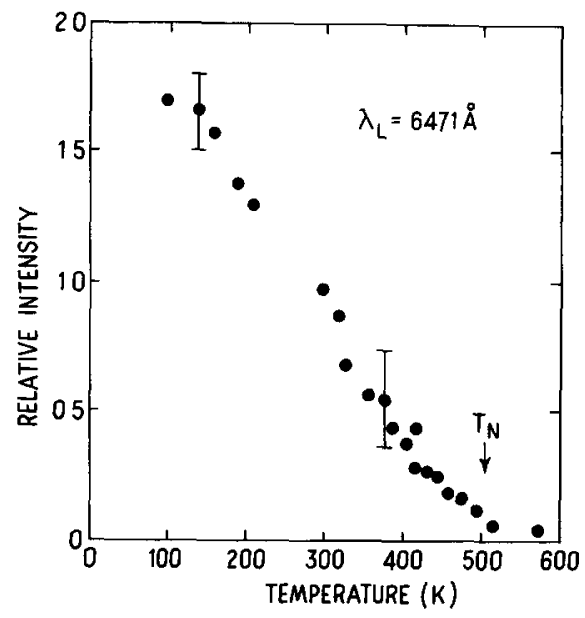

Fig. 2. Intensity of the two-magnon peak, measured relative to the intensity of the $616 \mathrm{~cm}^{-1}-A_{g}$ phonon, as a function of temperature. The arrow indicates the Nèel temperature.

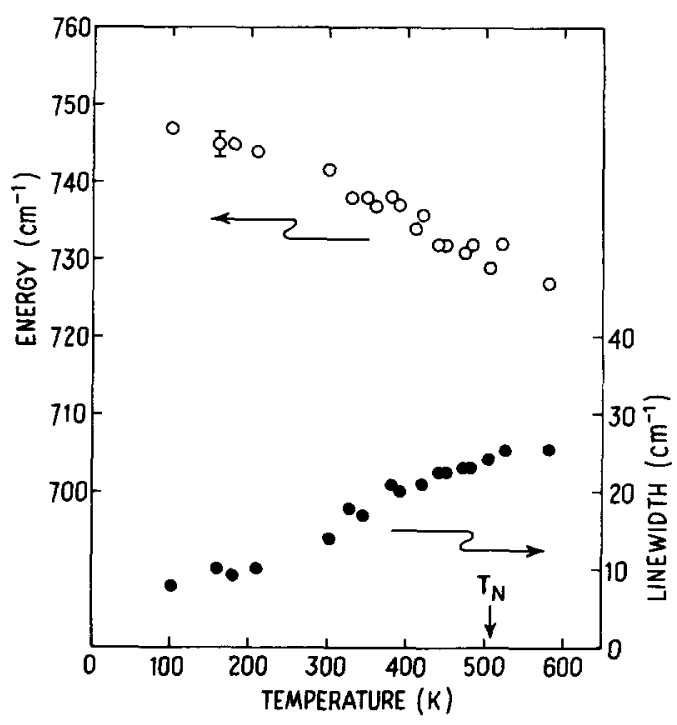

Fig. 3. Temperature dependence of the peak position (left ordinate) and the linewidth (right ordinate) of the two-magnon band. The Neel temperature is indicated by an arrow.

those two spins associated with pairs of Fe-ions $2.81 \AA$ apart, for which the coupling to the further distant nn is likely to be substantially reduced [4].

Finally, we consider the temperature dependence of the scattering intensity in both $\mathrm{Fe}_{3} \mathrm{BO}_{6}$ and $\alpha-\mathrm{Fe}_{2} \mathrm{O}_{3}$. There are few theoretical predictions concerning the high-temperature behavior of two-magnon spectra [11]. A calculation of four-spin static correlations at $T=\infty$ and $T=0$ indicates that [12]

$I_{\infty} / I_{0}=1 / 3(S+1)^{2}$. 
This prediction, valid only for two-sublattice antiferromagnets, appears to be in reasonable agreement with the few experimental results that are available $[11,12]$. Clearly, equation (1) cannot be applied to our data. We believe that the diminution of the scattering strength in $\mathrm{Fe}_{3} \mathrm{BO}_{6}$ and hematite relates to the thermal break-up of the spin clusters discussed above. The misalignment of just one of the spins in a cluster leads to a shift of its lowest excitation energy out of the narrow localized band, so that it will no longer contribute to the observed peak. The Raman intensity should reflect the probability of finding a cluster with the moments properly aligned and should therefore behave, at least qualitatively, in the manner which we observe. Calculations to develop this idea for the specific cases of $\mathrm{Fe}_{3} \mathrm{BO}_{6}$ and $\alpha-\mathrm{Fe}_{2} \mathrm{O}_{3}$ are currently underway.

Acknowledgements - We would like to thank R. Clarke for the use of his X-ray equipment. One of us (H.N.) gratefully acknowledges the support of CONACyT and Secretaria de Educación Pública de México through DGICSA. This work was performed under the auspices of the U.S. Army Research Office under contract No. DAAG-29-82-K0057, and Research Corporation.

\section{REFERENCES}

1. R. Wolfe, R.D. Pierce, M. Eibschutz \& J.W. Nielsen, Solid State Commun. 7, 949 (1969).

2. J.G. White, A. Miller \& R.E. Nielsen, Acta Cryst. 16, 849 (1963).

3. M. Hirano, T. Okuda, T. Tsushima, S. Umemura, K. Kohn \& S. Nakamura, Solid State Commun. 15, 1129 (1974); C. Vorgt \& D. Bonnenberg, Physica 80B, 439 (1975).

4. B. Andlauer \& R. Diehl, Physica 89b, 50 (1977).

5. See, for example, D.L. Rousseau, R.P. Bauman \& S.P.S. Porto, J. Raman Spectrosc. 10, 253 (1981).

6. See, e.g., W. Hayes \& R. London, Scattering of Light by Crystals, Chapter 6. Wiley-Interscience, New York (1978).

7. T.P. Martin, R. Merlin, D.R. Huffman \& M. Cardona, Solid State Commun. 22, 565 (1977).

8. T.R. Hart, S.B. Adams \& H. Temkin, Light Scattering in Solids (Edited by M. Balkansky, R.C.C. Leite \& S.P.S. Porto), p. 259. Flammarion, Paris (1976).

9. E.J. Samuelson \& G. Shirane, Phys. Status Solidi 42, 241 (1970).

10. E.J. Samuelson, Physica 43, 353 (1969).

11. U. Balucani \& V. Tognetti, Nuovo Cimento 6, 39 (1976).

12. W.J. Brya \& P.M. Richards, Phys. Rev. B9, 2244 (1974). 